\title{
Outcomes of Intramedullary Nailing with Cerclage Wiring in Subtrochanteric Femoral Fractures
}

\author{
Nisarg J Mehta ${ }^{1}$, Tom Goldsmith² ${ }^{2}$ Alice Lacey ${ }^{3}$, Gautam Reddy ${ }^{4}$, Veenesh Selvaratnam ${ }^{5}$, Muthukrishnan Ramakrishnan ${ }^{6}$
}

\begin{abstract}
Aims: The aim of this study was to compare the outcomes of closed reduction against open reduction with cerclage wires in patients with subtrochanteric fractures treated with intramedullary nailing (IMN).

Materials and methods: We identified 141 patients who had an IMN over a 4-year period. They were classified into three groups based on fracture pattern and whether open or closed reduction was performed. Type I was a transverse fracture, type II, a spiral fracture with an intact posterior and medial wall in the proximal fragment, and a type III fracture without intact posterior or medial walls. The primary outcome measure was a revision surgery for implant failure. Secondary outcome measures were related to fracture reduction and radiological union scores of the hip (RUSH).

Results: There were 35 patients who had a type I fracture, 26 patients with a type II fracture, and 80 patients with a type III fracture. The mean follow-up was 7 months. Closed reduction in type III fractures was associated with a significantly increased risk of mechanical complications $(p=0.005)$ and unplanned returns to theatre for implant failure $(p=0.04)$ as compared to open reduction. Open reduction in type III fractures was associated with a significantly higher mean RUSH scores $(p=0.0006)$. There was no significant difference in mean operative time between open and closed reduction in type III fractures $(p=0.12)$.

Conclusion: We recommend open reduction with cerclage wiring in type III subtrochanteric fractures in order to reduce the risk of implant failure, nonunion, and need for further surgery.

Keywords: Cerclage wiring, Intramedullary nailing, Subtrochanteric fracture.

Strategies in Trauma and Limb Reconstruction (2019): 10.5005/jp-journals-10080-1423
\end{abstract}

\section{INTRODUCTION}

Subtrochanteric fractures are challenging problems to treat operatively because of various anatomical and biomechanical factors. ${ }^{1}$ Poor vascularity and high tensile and compressive forces contribute to the challenge of fracture healing. ${ }^{2}$ Cephalomedullary nails are the mainstay of treatment. However, problems in obtaining anatomical reduction can arise in the presence of a short proximal fragment with a flexion, abduction, and external rotation deformity. ${ }^{1,3}$ If anatomical reduction is not achieved, the wide proximal femoral canal limits indirect reduction techniques and can lead to malalignment, which is a recognized risk factor for nonunion and implant failure. ${ }^{1,3}$ Nonunion rates of up to $7 \%$ have been reported in cases of malalignment. ${ }^{4,5}$ Anatomical reduction can be achieved by the intraoperative use of cerclage wires to hold and maintain reduction, which is associated with significantly reduced nonunion rates of $<2 \% .{ }^{4-6}$ This may add to the overall construct stability, improve the quality of reduction, and improve the load sharing properties of the intramedullary device. ${ }^{7}$

There are numerous classification systems of subtrochanteric fractures, but there is no consensus to guide surgeons when to perform open reduction intraoperatively when closed reduction fails. ${ }^{8}$ The purpose of this study was to compare the outcomes of closed reduction against open reduction with cerclage wires in patients with traumatic subtrochanteric fractures treated with IMN. Based on the results, we aim to also propose a new classification system to dictate management.

\section{Materials and Methods}

This is a retrospective review of all patients with traumatic subtrochanteric fractures presenting to a university teaching hospital between 2011 and 2015 and treated by a proximal femoral
1,4,6 Wirral University Teaching Hospital, Wirral, UK

${ }^{2}$ Northern General Hospital, Sheffield, UK

${ }^{3}$ Wrexham Maelor Hospital, Wrexham, Wales, UK

${ }^{5}$ Exeter Hip Unit, Royal Devon and Exeter Hospital, Exeter, UK

Corresponding Author: Nisarg J Mehta, Wirral University Teaching Hospital, Wirral, UK, Phone: +91 7525048186, e-mail: njmehta28@ yahoo.co.uk

How to cite this article: Mehta NJ, Goldsmith T, Lacey A, et al. Outcomes of Intramedullary Nailing with Cerclage Wiring in Subtrochanteric Femoral Fractures. Strategies Trauma Limb Reconstr 2019;14(1):29-33.

Source of support: Nil

Conflict of interest: None

nail antirotation (PFNA) (Synthes UK). ${ }^{9}$ All nails had standard distal locking screws inserted. Pathological fractures, those associated with bisphosphonate use, intertrochanteric, and segmental fractures were excluded from this study. A subtrochanteric fracture was defined as an injury in which the main fracture line was located within the area from the distal border of the lesser trochanter to a distance of $5 \mathrm{~cm}$ distally. ${ }^{1,3}$ After a review of current classification systems, and finding them unsuitable for the purposes and objectives of this study, a simpler 3-tier classification system of subtrochanteric fractures was proposed by the senior author (Fig. 1). Type I indicated a transverse fracture, type II was a spiral fracture with an intact posterior and medial wall in the proximal fragment, and a type III fracture was a comminuted fracture with no posterior or medial wall integrity.

Data were collected over a 4-year period (2011-2015). Patients with subtrochanteric fractures were classified into type I, II, or III fractures based on radiographs by two independent senior clinicians. The cases were then divided into two groups based on whether treatment had 


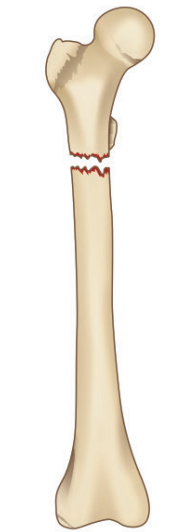

Type I

Transverse fracture
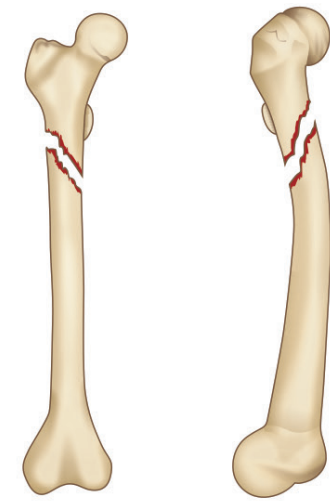

Type II

Spiral fracture, intact posterior and medial wall
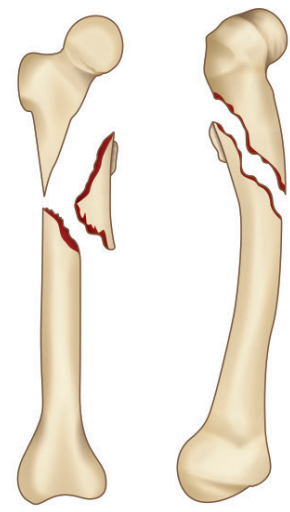

Type III

Comminuted fracture, no posterior or medial wall

Fig. 1: A new classification system for subtrochanteric fractures

been done by open reduction with cerclage wires or closed reduction using a traction table. Clinical and radiographic data collected included transfusion requirements, complications, operative time, American Society of Anaesthesiology (ASA) score, and the tip-apex distance. Mobilization was full weight bearing postoperatively. Thromboprophylaxis with enoxaparin was administered during the stay in hospital and up to 28 days postoperatively.

The primary outcome measure was revision surgery for failure; this was defined lag screw cut out, distal locking screw failure, and nonunion. Secondary outcome measures were related to the quality of fracture reduction and validated RUSH. The RUSH provides a standardized radiographic assessment of hip fracture healing based on the absence or presence of bridging callus and the appearance of the fracture line and is a reliable method for assessing fracture healing. ${ }^{10}$ The RUSH was calculated on 39 patients by 2 independent clinicians (blinded) at separate intervals on anteroposterior (AP) and lateral radiographs taken 6 months post injury. Nonunion was defined as the absence of radiological healing 6 months post surgery. ${ }^{11}$ Fracture reduction was also assessed by two blinded independent clinicians at separate intervals and was measured on antero-posterior and lateral radiographs. The quality of reduction was assessed from Baumgaertner et al.'s work: good was both cortical displacement $<4 \mathrm{~mm}$ and angulation $<10^{\circ}$; acceptable was either cortical displacement $<4 \mathrm{~mm}$ or angulation $<10^{\circ}$; and poor was cortical displacement $>4 \mathrm{~mm}$ and angulation $>10^{\circ} .{ }^{12}$

Statistical analysis was performed using GraphPad Prism version 6, GraphPad Software Inc., California, USA. Data were summarized using the mean and range for continuous and categorical data. Fisher's exact test was used for independent categorical data and Mann-Whitney $U$ test for continuous nonparametric data. Statistical significance was set at $p<0.05$.

\section{Results}

There were 141 cases of IMN for subtrochanteric fractures over the 4-year period. One hundred and two patients were lost to follow-up as patients with subtrochanteric fractures and hip fractures are not routinely reviewed postoperatively at our institution. There were 35 patients who had a type I fracture, 26 patients with a type II fracture, and 80 patients with a type III fracture.

Thirty-nine patients were identified who met the inclusion criteria and were followed-up for a mean of 7 months. Table 1 compares the demographic factors for the different fracture types.
Table 2 compares the mechanical complications, return to theatre, and the mean tip-apex distances for the different type of fractures treated by open or closed reduction. The three fracture types were matched evenly with respect to age and ASA grade. Closed reduction in type III fractures was associated with a significantly increased risk of mechanical complications compared to open reduction. In addition, a significantly higher proportion of patients with type III fractures treated by closed reduction returned to theatre for implant failure (Table 2). Open reduction in type III fractures was associated with a significantly higher mean RUSH value $(p=0.0006)$. There was no significant difference in mean operative time between open and closed reductions in type III fractures $(p=0.12)$. Table 3 lists the quality of reduction between open and closed reductions for types I to III fractures and the risk of implant failure. Open reduction was associated with a better quality of reduction graded as good compared to closed reduction in type II $(p=0.03)$ and type III fractures $(p<0.0001)$. In addition, poor reduction was associated with an increased risk of implant failure in type I $(p=0.017)$ and type III fractures $(p=0.02)$. Other factors such as the tip-apex distance had no significant effect on implant failure or return to theatre. Furthermore, there was no significance difference in the ages, ASA, transfusion requirements, and lengths of stay in types I to III fractures treated with open or closed reduction (Table 1).

\section{Discussion}

This study shows that the addition of cerclage wires achieved a better quality of reduction in type III subtrochanteric fractures. This, in turn, incurred no overall increase in mean operative time but was associated with a significantly reduced risk of mechanical complications requiring return to theatre. There are several classification systems for subtrochanteric fractures such as Seinsheimer ${ }^{13}$ and Fielding. ${ }^{14}$ None of these have reported any proven benefit in determining the choice of treatment and prognosis. ${ }^{8}$ This study has shown that the proposed three-tier classification can be used as a decision-making tool for subtrochanteric fractures and can guide the surgeon when to perform open reduction with cerclage wires. As a result of the findings here, we recommend open reduction with cerclage wires for all patients with a deficient medial or posterior wall type III fracture.

Our classification system is based upon the deforming forces that act on the proximal fracture fragment. This includes flexion and external rotation from iliopsoas, further external rotation from 
Intramedullary Nailing with Cerclage Wiring in Subtrochanteric Femoral Fractures

Table 1: Comparison of demographic and operative factors in patients with types I to III subtrochanteric fractures

\begin{tabular}{|c|c|c|c|c|c|c|c|}
\hline & Type IOR & Type ICR & Type II OR & Type /I CR & Type III OR & Type III CR & $p$ value \\
\hline Age & 75.5 (75-76) & 83.2 (64-99) & $85.4(67-95)$ & $87.1(69-98)$ & $81.6(60-96)$ & $83.9(61-95)$ & 0.19 \\
\hline Significance & \multicolumn{2}{|c|}{$p=0.11$} & \multicolumn{2}{|c|}{$p=0.51$} & \multicolumn{2}{|c|}{$p=0.21$} & \\
\hline ASA grade & $2(2)$ & $2.76(1-4)$ & $2.81(1-3)$ & $2.73(1-4)$ & $2.76(2-4)$ & $2.80(2-4)$ & 0.82 \\
\hline Significance & \multicolumn{2}{|c|}{$p=0.07$} & \multicolumn{2}{|c|}{$p=0.43$} & \multicolumn{2}{|c|}{$p=0.79$} & \\
\hline Operative time min & $109.0(96-122)$ & $97.6(54-184)$ & $122.2(80-185)$ & $76.5(37-119)$ & $105.1(57-220)$ & $93.3(55-183)$ & 0.0004 \\
\hline Significance & \multicolumn{2}{|c|}{$p=0.49$} & \multicolumn{2}{|c|}{$p=0.0004$} & \multicolumn{2}{|c|}{$p=0.12$} & \\
\hline RBC units transfused & $2.5(1-4)$ & $2.1(1-3)$ & $2.3(2-3)$ & $2.4(2-3)$ & $2.59(1-5)$ & $2.23(1-4)$ & 0.26 \\
\hline Significance & \multicolumn{2}{|c|}{$p=0.99$} & \multicolumn{2}{|c|}{$p=0.99$} & \multicolumn{2}{|c|}{$p=0.17$} & \\
\hline Drop in $\mathrm{Hb} \mathrm{g} / \mathrm{dL}$ & $52.5(29-76)$ & $25.2(0-56)$ & $35.2(5-49)$ & $25.3(8-50)$ & $29.5(5-70)$ & $29.3(3-57)$ & 0.21 \\
\hline Significance & \multicolumn{2}{|c|}{$p=0.18$} & \multicolumn{2}{|c|}{$p=0.14$} & \multicolumn{2}{|c|}{$p=0.79$} & \\
\hline RUSH score & $24.7(16-28)$ & $25(24-26)$ & $29.4(27-30)$ & $10(10)$ & $26.3(11-30)$ & $20.7(10-30)$ & 0.0006 \\
\hline Significance & \multicolumn{2}{|c|}{$p=0.75$} & \multicolumn{2}{|c|}{$p=0.047$} & \multicolumn{2}{|c|}{$p=0.022$} & \\
\hline Length of stay $d$ & $48.5(25-72)$ & $21.7(2-58)$ & $21.4(11-38)$ & $22.4(2-47)$ & $39.7(4-370)$ & $30.9(4-106)$ & 0.38 \\
\hline Significance & \multicolumn{2}{|c|}{$p=0.19$} & \multicolumn{2}{|c|}{$p=0.84$} & \multicolumn{2}{|c|}{$p=0.67$} & \\
\hline
\end{tabular}

Table 2: Comparison of mechanical complications, mean tip-apex distance, $\mathrm{mm}$, and return to theater in types I to III subtrochanteric fractures

\begin{tabular}{|c|c|c|c|c|c|c|c|c|c|}
\hline Classification & Reduction $n$ & $\begin{array}{l}\text { Mechanical } \\
\text { complications } n\end{array}$ & & Significance & Return to theatre & $n$ & Significance & TAD $\mathrm{mm}$ & Significance \\
\hline \multirow[t]{3}{*}{ Type I } & Open 2 & \multicolumn{2}{|c|}{ No complications } & $p=0.99$ & No surgery & 2 & $p=0.99$ & $\mathrm{~N} / \mathrm{A}$ & N/A \\
\hline & \multirow[t]{2}{*}{ Closed 33} & $\begin{array}{l}\text { Distal locking } \\
\text { screw failure }\end{array}$ & 1 & & No surgery & 1 & & $19.1,18.3-20$ & \\
\hline & & Nonunion & 1 & & Exchange nailing & 1 & & & \\
\hline \multirow[t]{2}{*}{ Type II } & Open 11 & $\begin{array}{l}\text { Distal locking } \\
\text { screw failure }\end{array}$ & 1 & $p=0.99$ & No surgery & 1 & $p=0.99$ & $14.5,4.5-14.5$ & $\mathrm{~N} / \mathrm{A}$ \\
\hline & Closed 15 & Nonunion & 1 & & Femoral plating & 1 & & $36.2,36.2-36.2$ & \\
\hline \multirow[t]{11}{*}{ Type III } & \multirow[t]{3}{*}{ Open 39} & \multirow{3}{*}{$\begin{array}{l}\text { Distal locking } \\
\text { screw failure }\end{array}$} & \multirow[t]{3}{*}{3} & $p=0.005$ & Nail removal & 1 & \multirow{3}{*}{$\begin{array}{l}p=0.04 \\
\text { NNT = 3.10, } \\
95 \% \text { Cl 1.6-7.6 }\end{array}$} & \multirow[t]{3}{*}{$34.07,9.5-60$} & \multirow[t]{3}{*}{$\mathrm{N} / \mathrm{A}$} \\
\hline & & & & $\mathrm{NNT}=2.5$ & Exchange nailing & 1 & & & \\
\hline & & & & $95 \%$ Cl 1.4-4.6 & No surgery & 1 & & & \\
\hline & \multirow[t]{8}{*}{ Closed 41} & \multirow[t]{5}{*}{$\begin{array}{l}\text { Lag screw cut } \\
\text { out }\end{array}$} & \multirow[t]{5}{*}{8} & & $\begin{array}{l}\text { Proximal femur } \\
\text { replacement }\end{array}$ & 1 & & \multirow[t]{8}{*}{$21.3,8.4-49.0$} & \multirow[t]{8}{*}{$p=0.44$} \\
\hline & & & & & Total hip replacement & 3 & & & \\
\hline & & & & & Removal of metal & 1 & & & \\
\hline & & & & & Exchange nailing & 1 & & & \\
\hline & & & & & No surgery & 2 & & & \\
\hline & & \multirow{2}{*}{\multicolumn{2}{|c|}{$\begin{array}{l}\text { Distal locking } \\
\text { screw failure }\end{array}$}} & & Total hip replacement & 1 & & & \\
\hline & & & & & $\begin{array}{l}\text { Removal of metal- } \\
\text { work }\end{array}$ & 4 & & & \\
\hline & & Nonunion & 1 & & Exchange nailing & 1 & & & \\
\hline
\end{tabular}

the short external rotators, abduction from the gluteus medius and minimus, and, lastly, adduction and shortening of the shaft from the hamstrings and adductors ${ }^{15,16}$ (Fig. 2). The subtrochanteric region is also eccentrically loaded and the compressive medial forces outweigh the tensile lateral forces. ${ }^{17}$ Therefore, any implant can fail in the presence of malreduction. In type I and II fractures, the long posterior wall will confer stability by neutralizing these deforming forces after nail insertion with closed reduction. Open reduction is required in type III fractures owing to the deficiency in the posterior and medial buttress. Nail insertion following closed reduction in such unstable fractures can result in further displacement of the proximal fragment which is associated with an increased risk of implant failure. By applying a cerclage wire intraoperatively in type III fractures, anatomical reduction is achieved through contact between bone ends; this adds to the overall stability of the implant-fracture construct and reduces the risk of malalignment and implant failure. ${ }^{4}$

There was an increased risk of implant failure and nonunion in patients with type III fractures who were treated with closed reduction because of malreduction and malalignment. The importance of anatomical reduction in such fractures is highlighted in this series and emphasized by findings of other clinical and biomechanical studies. ${ }^{1,18-20}$ Distal locking screw failure indicates instability of the overall mechanical construct. ${ }^{11}$ Giannoudis et al. demonstrated that distal locking screw failure and subsequent breakage of the nail was the standard mode of failure of cephalomedullary nails and recommended that early breakage of distal locking screws in a symptomatic patient should be utilized as a predictor of pending failure. ${ }^{11}$ There were similar findings in this study as $48 \%(10 / 21)$ of patients with mechanical complications 
Table 3: Comparison of the quality of reduction and incidence of implant failure (IF) in types I to III subtrochanteric fractures

\begin{tabular}{|c|c|c|c|c|c|c|c|}
\hline \multirow[b]{2}{*}{ Fracture type } & \multicolumn{7}{|c|}{ Quality of reduction, implant failure, IF and significance, $p$} \\
\hline & Good & IF $n$ & Acceptable & $I F n$ & Poor & $I F n$ & $\begin{array}{l}\text { Fisher's exact } \\
\text { test } p\end{array}$ \\
\hline Type IOR & 1 & 0 & 0 & 0 & 1 & 0 & 0.017 \\
\hline Type I CR & 16 & 0 & 13 & 0 & 4 & 2 & \\
\hline Type II OR & 9 & 0 & 2 & 1 & 0 & 0 & 0.35 \\
\hline Type II CR & 5 & 0 & 5 & 0 & 5 & 1 & \\
\hline Type III OR & 33 & 0 & 3 & 2 & 3 & 1 & 0.02 \\
\hline Type III CR & 2 & 0 & 10 & 4 & 29 & 10 & \\
\hline
\end{tabular}

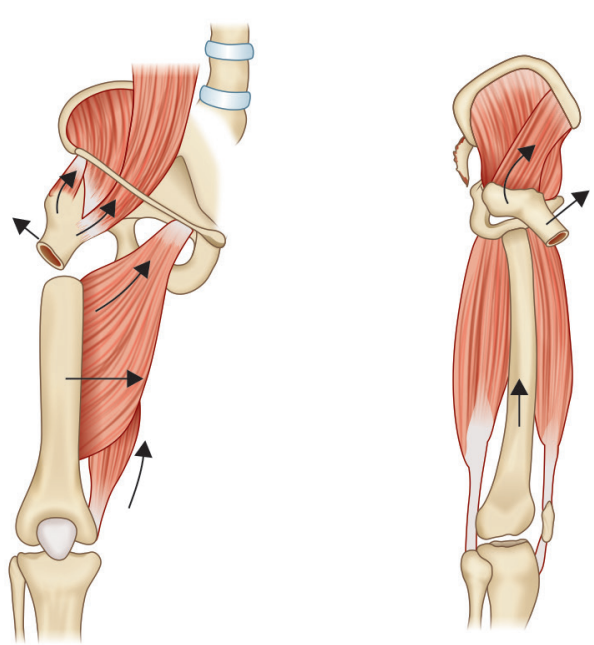

Fig. 2: Deforming forces acting on the subtrochanteric region of the proximal femur from the iliopsoas, gluteal muscles, hamstrings, adductors, and short external rotators resulting in shortening with a flexion, abduction, and external rotation deformity of the proximal fragment

presented with distal locking bolt failure. Seventy percent of these patients underwent removal of metal or revision surgery for implant failure. ${ }^{2}$ We recommend that all patients with subtrochanteric fractures should be followed up closely in the outpatient clinic until radiological union.

Concern over the use of cerclage wires has centered on the impairment of periosteal blood supply to the fracture site which can lead to nonunion. Recent cadaveric studies have demonstrated that the vascular supply to the periosteum is circumferential rather than longitudinal with multiple musculo-periosteal vessels supplying this layer. Therefore, one or two well-spaced cerclage wires should have little to no effect on the periosteal blood supply. ${ }^{21,22}$ Although recent studies have reported good outcomes of cerclage wiring in subtrochanteric fractures, care must be taken when passaging wires; various authors have reported major complications such as femoral artery and vein injury. ${ }^{23}$

There are limitations to this study. As with all retrospective studies, there was no control over follow-up and there may be an under-reporting of complications that may have presented to other hospitals. In addition, long-term follow-up did not occur for most patients. This is because fractures of the neck of femur and subtrochanteric region are not followed up in our institution for extended periods. These patients are often frail, and this fracture carries a high mortality for this group. In addition, whilst our classification system has been shown here to guide surgeons as to when to perform an open reduction in some subtrochanteric fractures, it is not yet validated and designed from a relatively small number of patients. Validity studies are being undertaken currently at the author's institution by testing the interobserver and intraobserver reliabilities via a test-retest analysis.

\section{Conclusion}

We propose a three-tier classification of subtrochanteric fractures that can guide the operating surgeon as to when to an open reduction can influence outcome. We recommend achieving and maintaining anatomical reduction by open reduction with cerclage wires in type III subtrochanteric fractures which have a short posterior wall and deficient medial buttress. Use of cerclage wires in such cases is associated with a significantly better quality of reduction and has been shown to reduce the risk of implant failure, nonunion, and need for further surgery.

\section{References}

1. Hoskins WA, Bingham R, Joseph S, et al. Subtrochanteric fracture: The effect of cerclage wire on fracture reduction and outcome. Injury 2015;46(10):1992-1995. DOI: 10.1016/j.injury.2015.07.001.

2. Lundy DW. Subtrochanteric femoral fractures. J Am Acad Orthop Surg 2007;15(11):663-671. DOI: 10.5435/00124635-200711000-00005.

3. Craig N, Sivaji S, Mafulli N. Subtrochanteric fractures. A review of treatment options. Bull Hosp Jt Dis 2001;60(1):35-46.

4. Tomás J, Teixidor J, Batalla L, et al. Subtrochanteric Fractures: Treatment With Cerclage Wire and Long Intramedullary Nail. J Orthop Trauma 2013;27(7):157-160. DOI: 10.1097/BOT.0b013e31826fc03f.

5. Robinson CM, Houshian S, Khan LA. Trochanteric-entry long cephalomedullary nailing of subtrochanteric fractures caused by low-energy trauma. J Bone Joint Surg Am 2005;87(10):2217-2226. DOI: 10.2106/JBJS.D.02898.

6. Afsari A, Liporace F, Lindvall E, et al. Clamp- assisted reduction of high subtrochanteric fractures of the femur. J Bone Joint Surg Am 2009;91(8):1913-1916. DOI: 10.2106/JBJS.H.01563.

7. Kim JW, Park KC, Oh JK, et al. Percutaneous cerclage wiring followed by intramedullary nailing for subtrochanteric femoral fractures: a technical note with clinical results. Arch Orthop Trauma Surg 2014;134(9):1227-1235. DOI: 10.1007/s00402-014-2023-7.

8. Loizou CL, McNamara I, Ahmed K, etal.Classification of subtrochanteric femoral fractures. Injury 2010;41(7):739-745. DOI: 10.1016/j. injury.2010.02.018.

9. UK Synthes. Proximal Femoral Nail Antirotation; 2016.

10. Frank T, Osterhoff G, Sprague S, et al. The radiographic union score for hip (RUSH) identifies radiographic nonunion of femoral neck fractures. Clin Orthop Relat Res 2016;474(6):1396-1404. DOI: 10.1007/ s11999-015-4680-4. 
11. Giannoudis PV, Ahmad MA, Mineo GV, et al. Subtrochanteric fracture non-unions with implant failure managed with the "Diamond" concept. Injury 2013;44(1):76-81. DOI: 10.1016/S0020-1383(13) 70017-2.

12. Baumgaertner MR, Curtin SL, Lindskog DM, et al. The value of the tip-apex distance in predicting failure of fixation of peritrochanterc fractures of the hip. J Bone Joint Surg Am 1995;77(7):1058-1064. DOI: 10.2106/00004623-199507000-00012.

13. Seinsheimer F. Subtrochanteric fractures of the femur. J Bone Joint Surg Am 1978;60(3):300-306. DOI: 10.2106/00004623-19786003000004.

14. Fielding JW. Subtrochanteric fractures. Clin Orthop Relat Res 1973;92:86-99. DOI: 10.1097/00003086-197305000-00008.

15. Hak D, Wu H, Dou C, et al. Challenges in Subtrochanteric Femur Fracture Management. Orthopaedics 2015;38(8):498-502. DOI: 10.3928/01477447-20150804-06

16. Gong J, Yang Y, Liu P, et al. PFNA with reduction assisted with pointed clamp and cable cerclagefor select subtrochanteric fractures of the femur. Int J Clin Exp Med 2016;9(2):2961-2968.

17. Bedi A, Toan L. Subtrochanteric femur fractures. Orthop Clin North Am 2004;35(4):473-483. DOI: 10.1016/j.ocl.2004.05.006.
18. Haidukewych GJ,BerryDJ.Nonunion of fractures of the subtrochanteric region of the femur. Clin Orthop Relat Res 2004;419:185-188. DOI: 10.1097/00003086-200402000-00030.

19. Beingessner DM, Scolaro JA, Orec RJ, et al. Open reduction and intramedullary stabilisation of subtrochanteric femur fractures: A retrospective study of 56 cases. Injury 2013;44(12):1910-1915. DOI: 10.1016/j.injury.2013.08.013.

20. Giannoudis PV, Einhorn TA, Marsh D. Fracture healing: the diamond concept. Injury 2007;38(Suppl 4):S3-S6. DOI: 10.1016/S00201383(08)70003-2.

21. Pazzaglia UE, Congiu T, Raspanti M, et al. Anatomy of the intracortical canal system. Scanning electron microscopy study in rabbit femur. Clin Orthop Relat Res 2009;467(9):2446-2456. DOI: 10.1007/s11999009-0806-x.

22. Kennedy M, Mitra A, Hierlihy TG, et al. Subtrochanteric hip fractures treated with cerclage cables and long cephalomedullary nails: a review of 17 consecutive cases over 2 years. Injury 2011;42(11):13171321. DOI: 10.1016/j.injury.2011.03.023.

23. Mehta V, Finna HA. Femoral artery and vein injury after cerclage wiring of the femur: a case report. J Arthroplasty 2005;20(6):811-814. DOI: $10.1016 /$ j.arth.2004.12.050 